\title{
Avaliação da Efetividade e Segurança do Protocolo de Infusão de Insulina de Yale para o Controle Glicêmico Intensivo*
}

\author{
Assessment of Effectiveness and Safety of Yale Insulin Infusion \\ Protocol in a Brazilian Medical and Surgical Intensive Care Unit
}

\author{
José Roberto Carvalho Diener', Carlos Eduardo Elias dos Prazeres², \\ Cilmar Mello da Rosa ${ }^{3}$, Urubatan Collaço Alberton ${ }^{4}$, Carla Cristina Souza Ramos 5 .
}

\section{RESUMO}

JUSTIFICATIVA E OBJETIVOS: O controle glicêmico intensivo ocupa lugar de destaque no manuseio dos pacientes críticos. O objetivo desde estudo foi avaliar a efetividade e a segurança do protocolo de insulinoterapia por via venosa de Yale nos pacientes críticos internados em unidade de terapia intensiva geral em hospital comunitário.

MÉTODO: Foi realizado um estudo retrospectivo e comparativo entre 2 coortes de pacientes críticos, antes e após a implantação do controle glicêmico intensivo. Os desfechos de interesse do estudo foram glicemia média durante o tratamento, tempo para atingir a faixa alvo de 80 a $140 \mathrm{mg} / \mathrm{dL}$, percentual de glicemia

1. Mestre em Medicina pela UFSC. Especialista em Medicina Intensiva pela AMIB. Médico Chefe do Serviço de Terapia Intensiva do Hospital de Caridade de Florianópolis, SC. Coordenador da Equipe de Terapia Nutricional do Hospital Universitário da UFSC.

2. Graduando do Curso de Medicina da Universidade Federal de Santa Catarina - UFSC

3. Especialista em Medicina Intensiva pela AMIB. Médico do Serviço de Terapia Intensiva e da Equipe de Terapia Nutricional do Hospital de Caridade de Florianópolis, SC.

4. Especialista em Medicina Intensiva pela AMIB. Médico do Serviço de Terapia Intensiva e do Serviço de Endocrinologia do Hospital de Caridade de Florianópolis, SC.

5. Especialista em Enfermagem Intensiva e Emergência pela UNISUL. Enfermeira Chefe do Serviço de Terapia Intensiva do Hospital de Caridade de Florianópolis, SC.

*Recebido do Serviço de Terapia Intensiva do Hospital de Caridade de Florianópolis, SC

Apresentado em 31 de julho de 2006

Aceito para publicação em 06 de setembro

Endereço para correspondência:

Dr. José Roberto Carvalho Diener

Rua Menino Deus 376 - Centro

88020-210 Florianópolis, SC

Fone: 48-3221-7521 / Fax: 48-3221-7589

E-mail: jrdiener@hotmail.com

(C)Associação de Medicina Intensiva Brasileira, 2006 dentro desta faixa e incidência de hipoglicemia.

RESULTADOS: Foram estudados 112 pacientes, divididos em dois grupos. Sessenta pacientes constituíram o grupo controle (GC) e 52 o grupo protocolo (GP). A glicemia média no GP foi de 131,2 $\pm 14,7 \mathrm{mg} / \mathrm{dL}$ versus $181,7 \pm 36,1 \mathrm{mg} / \mathrm{dL}$ no GC. Os pacientes no GP alcançaram a faixa alvo mais rápido [mediana $7 \mathrm{~h}$ (4 - 10h) versus mediana $96 \mathrm{~h}(46-278 \mathrm{~h})]$ no GC. O percentual de glicemia dentro da faixa-alvo foi de $65 \%$ no GP e de $32 \%$ no GC. Não houve diferença estatística significativa na incidência de hipoglicemia grave; 4 pacientes no GP versus 2 pacientes no GC.

CONCLUSÕES: O protocolo de insulinoterapia por via venosa contínua de Yale, mostrou-se efetivo e seguro para o manuseio do controle glicêmico em unidade de terapia intensiva que atende pacientes clínicos e cirúrgicos.

Unitermos: Doença crítica, hiperglicemia, insulina, protocolos, terapia intensiva.

\section{SUMMARY}

BACKGROUND AND OBJECTIVES: Actually tight glycemic control is a major concern in critical care. The objective of this study was to evaluate effectiveness and safety of Yale insulin infusion protocol in a Brazilian medical and surgical intensive care unit.

METHODS: Retrospective, before-after cohort study. Selected end-points were mean blood glucose levels, time-to-reach target range of $80-140 \mathrm{mg} / \mathrm{dL}$, and percent of blood glucose in target range and hypoglycemia incidence.

RESULTS: Were studied 112 patients: 60 in control group (CG) and 52 in protocol group (PG). Bedside blood glucose was measured 5392 times for a mean value of $131.2 \pm 14.7 \mathrm{mg} / \mathrm{dL}$ in the PG versus $2485 \mathrm{ti}-$ mes for a mean value of $181.7 \pm 36.1 \mathrm{mg} / \mathrm{dL}$ in the CG. Blood glucose values were in the target range $65 \%$ and 
$32 \%$ of the times, respectively for $P G$ and $C G$ groups $(p<0.001)$. The median time to reach glucose target range was $7 \mathrm{~h}$ (range $4-10 \mathrm{~h}$ ) for $\mathrm{PG}$ and $96 \mathrm{hr}$ (range $46-278 h)$ for $C G(p<0.001)$. Incidence of severe hypoglycemia did not reach difference statistically significant: 4 patients in $P G$ versus 2 patients in $C G$.

CONCLUSIONS: Yale insulin infusion protocol was effective and safe to improve blood glucose control in a Brazilian medical and surgical intensive care unit.

Key Words: Critical illness, hyperglycemia, insulin, intensive care, protocols.

\section{INTRODUÇÃO}

A hiperglicemia ocorre com freqüência nos pacientes críticos diabéticos e não diabéticos. Admite-se que ela seja causada por aumento da gliconeogênese e por resistência periférica à ação da insulina em resposta à secreção de hormônios contra-regulatórios e de citocinas inflamatórias ${ }^{1}$.

Ela aceita previamente como uma resposta apropriada e mesmo benéfica ao estresse, atualmente a hiperglicemia é reconhecida como um fator de risco para evolução clínica desfavorável e aumento de mortalidade $^{2}$. Em situações agudas como infarto agudo do miocárdio, cirurgia cardiovascular, acidente vascular encefálico e traumatismo craniano, a glicemia elevada se associa à pior prognóstico ${ }^{3-6}$. Acredita-se que os efeitos deletérios estejam relacionados principalmente a depressão da função imune e a indução de um estado pró-inflamatório e pró-coagulante ${ }^{7-9}$.

A atitude frente à hiperglicemia no paciente crítico se modificou substancialmente após a publicação do estudo de Van den Berghe e col..$^{10} \mathrm{em} 2001$. Esse estudo demonstrou que o controle rigoroso da glicemia entre 80 e 110 $\mathrm{mg} / \mathrm{dL}$, utilizando infusão venosa contínua de insulina, reduziu significativamente a mortalidade e a incidência de complicações, num grupo de 1548 pacientes predominantemente cirúrgicos ${ }^{10}$. O controle estrito da glicemia passou a ser considerada conduta ideal ${ }^{11}$ e a utilização da insulinoterapia venosa contínua generalizou-se nas unidades de terapia intensiva em todos os países.

O Colégio Americano de Endocrinologia ${ }^{11}$ recomenda manter a glicemia abaixo de $110 \mathrm{mg} / \mathrm{dL}$ em todos os pacientes críticos como proposto por Van den Berghe e col. ${ }^{10}$. Outras diretrizes recomendam insulinoterapia venosa contínua só para glicemias acima de 140 a 150 $\mathrm{mg} / \mathrm{dL}$ por considerar que este nível reduz o risco de hipoglicemia e é uma proposta mais realista fora dos ambientes de pesquisa clínica ${ }^{12-14}$.
Independentemente da faixa glicêmica escolhida é fundamental para a implementação do controle estrito da glicemia a utilização de um protocolo de insulinoterapia que permita manter a glicemia dentro da faixa selecionada e que evite ou reduza a ocorrência de hipoglicemia. $\mathrm{Na}$ literatura existem vários protocolos descritos com diferentes estratégias e recomendações ${ }^{10,15-20}$.

Este estudo teve por objetivos avaliar a efetividade e a segurança do protocolo de insulinoterapia venosa de Yale $^{18}$, empregado para manter a glicemia entre 80 e $140 \mathrm{mg} / \mathrm{dL}$, em pacientes críticos internados em unidade de terapia intensiva geral.

\section{MÉTODO}

O estudo foi realizado no Serviço de Terapia Intensiva do Hospital de Caridade de Florianópolis, hospital comunitário geral. A UTI dispõe de sete leitos e atende em média 600 pacientes adultos clínicos e cirúrgicos por ano. $\mathrm{O}$ atendimento médico é prestado por intensivistas diaristas e plantonistas. O serviço de enfermagem é coordenado por enfermeiro-chefe e cada turno de atendimento conta com um enfermeiro assistencial e seis técnicos de enfermagem.

O desenho do estudo foi retrospectivo, comparativo, entre coortes, antes e após a implantação do controle glicêmico estrito. A Comissão de Ética dispensou a assinatura do termo de consentimento informado por tratar-se de coleta retrospectiva de dados sobre prática terapêutica, já em uso na UTI desde outubro de 2004. Foram incluídos no estudo 112 pacientes, divididos em dois grupos. Sessenta pacientes constituíram o grupo controle (GC) e 52 o grupo protocolo (GP). No GP foram incluídos os pacientes internados na UTI, entre dezembro de 2004 a outubro de 2005 que receberam insulina venosa contínua para manter a glicemia na faixa de 80 a $140 \mathrm{mg} / \mathrm{dL}$, guiada pelo protocolo de Yale ${ }^{18}$. Este protocolo, descrito por Goldberg e col., exige mensurações inicialmente horárias de glicemia e foi desenvolvido para ser controlado pela equipe de enfermagem. Após a decisão médica de iniciar insulinoterapia intensiva as determinações de glicemia, capilar ou arterial, foram realizadas à beira do leito com glicosímetro portátil Accu-Check Advantage Roche ${ }^{\circledR}$ e a sua taxa de infusão foi modificada de acordo com as instruções detalhadas pelo protocolo, conforme descrito no anexo 1. A insulinoterapia intensiva, neste período, foi recomendada para glicemias $\geq 200 \mathrm{mg} / \mathrm{dL}$, porém não houve uma exigência rigorosa para iniciar somente acima deste valor. A insulina regular, diluída em solução fisiológica na con- 
centração de $1 \mathrm{U} / \mathrm{mL}$, foi administrada em bomba perfusora B. Braun, empregando-se seringa de $50 \mathrm{~mL}$.

No GC foram incluídos os pacientes internados na UTI, um ano antes da implantação do protocolo, dezembro de 2002 a outubro de 2003, que apresentaram pelo menos 1 medida de glicemia $\geq 200 \mathrm{mg} / \mathrm{dL}$ e que receberam tratamento com insulina por via subcutânea ou venosa por mais de 24 horas. Neste período a administração de insulina subcutânea ou venosa era realizada conforme a orientação do médico intensivista do turno, sem haver um protocolo que padronizasse o controle glicêmico.

Foram coletados do prontuário os dados referentes ao sexo, idade, peso, razão para admissão na UTI, história de diabete melito, escore APACHE II e glicemia no início do tratamento. Registrou-se o uso de fármacos vasoativos, nutrição parenteral ou enteral, corticoterapia e ventilação mecânica. Foram anotados as medidas de glicemia capilar ou arterial e o tempo necessário para atingir a faixa terapêutica de 80 a $140 \mathrm{mg} / \mathrm{dL}$. No GP registrou-se a taxa de infusão horária de insulina durante o período de sua utilização.

Definiu-se como efetividade o poder do protocolo em atingir e manter a faixa glicêmica estabelecida de 80 a $140 \mathrm{mg} / \mathrm{dL}$. A segurança foi avaliada em relação ao número de episódios de hipoglicemia ocorridos. Considerou-se como hipoglicemia moderada valores de glicemia capilar $\leq 60 \mathrm{mg} / \mathrm{dL}$ e hipoglicemia grave valores $\leq 40 \mathrm{mg} / \mathrm{dL}$.

A análise estatística foi realizada com o programa Epiinfo 6.04. As variáveis contínuas foram comparadas utilizando o teste $t$ de Student e as proporções empregando o teste do Qui-quadrado. O tempo para a glicemia diminuir até $\leq 140 \mathrm{mg} / \mathrm{dL}$ foi comparado entre os 2 grupos utilizando-se a curva de sobrevida de Kaplan-Meier com o teste de log-rank do pacote estatístico SPSS versão 8.0. Valores de $p<0,05$ foram considerados estatisticamente significativos para todos os testes.

\section{RESULTADOS}

Foram incluídos 112 pacientes, 60 no GC e 52 no GP, representando 459 e 423 dias-pacientes. As características dos 2 grupos estão demonstradas na tabela 1 . Não houve diferença significativa quanto ao sexo, idade, peso, admissão clínica ou cirúrgica, diagnóstico de diabete melito, uso de nutrição parenteral e corticosteróides. O escore de gravidade APACHE II foi significativo maior no GP e também houve significativamente mais uso de vasopressores e nutrição enteral neste grupo. O GC apresentou média glicêmica mais elevada no início da insulinoterapia.

Tabela 1 - Características dos Grupos Estudados

\begin{tabular}{lccc}
\hline Características & GC $(n=60)$ & GP $(n=52)$ & Valor de p \\
\hline Sexo masculino & $28(46,7 \%)$ & $27(51,9 \%)$ & \\
Idade * & $61,38 \pm 13,84$ & $62,32 \pm 16,9$ & \\
Peso $(\mathrm{kg}){ }^{*}$ & $75,06 \pm 16,82$ & $73,82 \pm 15,4$ & \\
APACHE II * & $19,48 \pm 7,45$ & $22,88 \pm 8,14$ & $<0,05$ \\
Diabete melito & $24(40 \%)$ & $15(29 \%)$ & \\
Glicemia inicial mg/ $257,88 \pm 65,73$ & $212,71 \pm 68,91$ & $<0,001$ \\
dL * & & & \\
Categoria de admis- & & & \\
são & & & \\
$\quad$ Clínico & $41(68,3 \%)$ & $37(71,2 \%)$ & \\
$\quad$ Cirúrgico & $19(31,7 \%)$ & $15(28,8 \%)$ & \\
Intervenções & & & \\
$\quad$ Vasopressores & $23(38 \%)$ & $32(62 \%)$ & $<0,05$ \\
$\quad$ Nutrição enteral & $18(30 \%)$ & $33(63 \%)$ & $<0,001$ \\
$\quad$ Nutrição parenteral & $10(17 \%)$ & $5(9,6 \%)$ & \\
$\quad$ Corticosteróides & $31(52 \%)$ & $31(60 \%)$ & \\
\hline
\end{tabular}

*Valores expressos em Média \pm DP

Realizaram-se 2485 mensurações de glicemia capilar ou arterial no GC e 5392 no GP. A média de determinações diárias foi de 5,4 $\pm 1,2$ no GC e 14,8 \pm 4,2 no GP. $\mathrm{O}$ GP alcançou a faixa-alvo de 80 a $140 \mathrm{mg} / \mathrm{dL}$ significativamente mais rápido como mostra a análise da curva de sobrevida de Kaplan-Meier na figura 1 ( $p<$ 0,001 pelo teste do log-rank). A mediana do tempo necessário para alcançar a faixa foi de 7 horas (intervalo 25\%-75\% de 4-10 horas) no GP e de 96 horas (intervalo $25 \%-75 \%$ de $46-278$ horas) no GC. Todos os pacientes do GP atingiram a faixa enquanto que no GC só 32 pacientes a alcançaram.

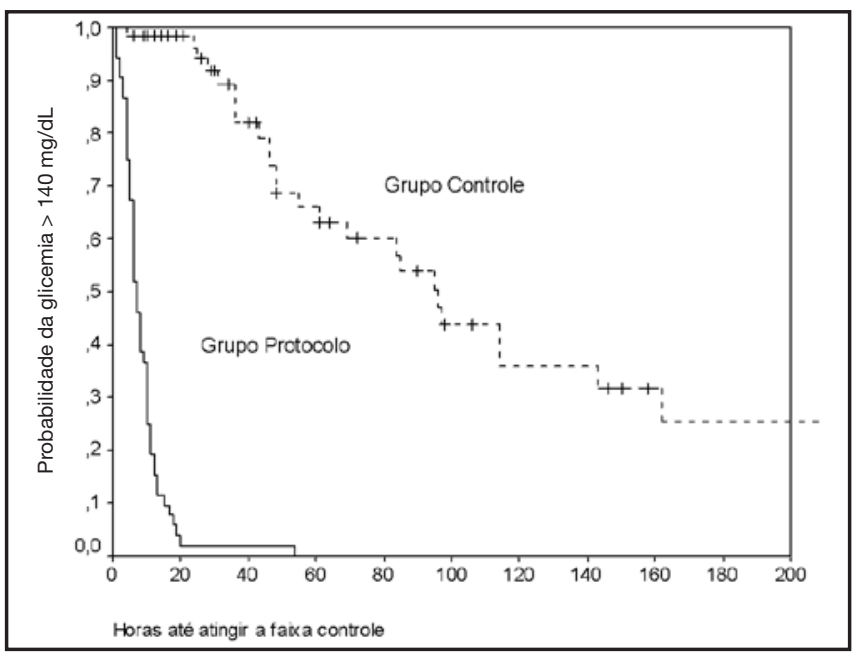

Figura 1 - Curva de Kaplan-Meier do Tempo (horas) para atingir o Controle Glicêmico nos Grupos Estudados.

As linhas verticais indicam os pacientes censurados por não atingir a faixa controle. 
A mediana do tempo de uso de insulina foi de 97 horas no GC e de 156 horas no GP. A infusão horária média de insulina por via venosa no GP foi de 2,2 $\pm 0,1$ (mediana 2) unidades.

Durante o tratamento a média glicêmica no GP foi de $131,2 \pm 14,7 \mathrm{mg} / \mathrm{dL}$ (mediana $123 \mathrm{mg} / \mathrm{dL}$ ) e no $\mathrm{GC}$ $181,7 \pm 36,1 \mathrm{mg} / \mathrm{dL}$ (mediana $166 \mathrm{mg} / \mathrm{dL}$ ). As médias dos valores glicêmicos diários dos 2 grupos, durante os 7 primeiros dias de tratamento, apresentaram diferença estatística significativa $(p<0,001)$, conforme demonstrado na figura 2 .

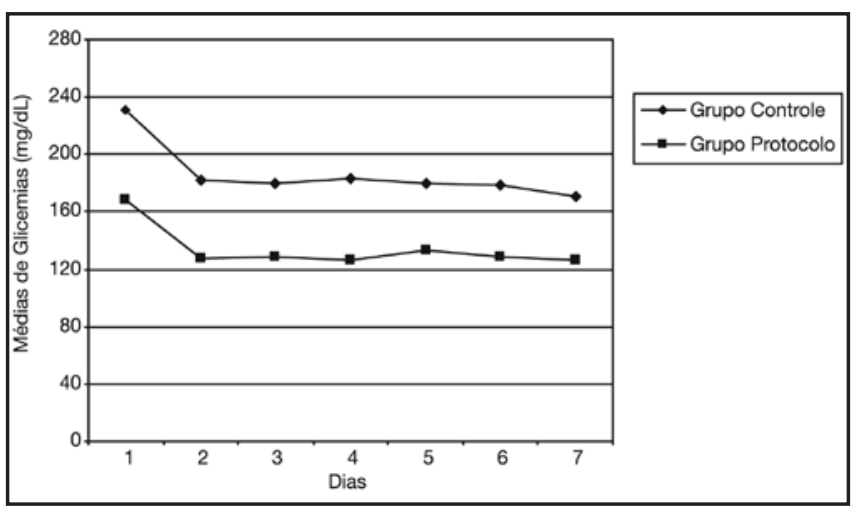

Figura 2 - Glicemias Médias durante os Sete Primeiros Dias de uso de Insulina nos Grupos Estudados

No GP das 5392 glicemias realizadas à beira do leito, 3527 (65\%) se situaram entre 80 e $140 \mathrm{mg} / \mathrm{dL}, 1597$ (30\%) acima e 266 (5\%) abaixo desta faixa. No GC, das 2485 mensurações, 799 (32\%) ficaram entre 80 e 140 $\mathrm{mg} / \mathrm{dL}, 1619$ (65\%) ficaram acima e 68 (2,7\%) abaixo, conforme demonstrado na tabela 2.

Ocorreram 44 episódios de hipoglicemia moderada em 18 pacientes e 4 episódios de hipoglicemia grave em 4 pacientes distintos no GP. Já no GC observaram-se 19 episódios de hipoglicemia moderada em 11 pacientes e três episódios de hipoglicemia grave em dois pacientes. Quando comparado o total de pacientes que desenvolveram hipoglicemia, houve diferença estatisticamente significativa entre os dois grupos. No GC 13 pacientes e GP 22 pacientes desenvolveram hipoglicemia, respectivamente $(p<0,05)$. Não houve diferença estatística significativa no número de pacientes com hipoglicemia grave; foram 2 pacientes no GC e 4 no GP. Não se observou nenhum evento clínico adverso grave relacionado aos episódios hipoglicêmicos.

\section{DISCUSSÃO}

Nos últimos anos o controle glicêmico intensivo, obtido pela infusão venosa contínua de insulina, passou a ocupar lugar de destaque no manuseio dos pacientes críticos. Alguns autores chegam a citá-lo como o maior avanço ocorrido na terapia intensiva desde a introdução da reanimação volêmica e da ventilação mecânica com pressão expiratória positiva ${ }^{21}$. A premissa é de que a manutenção da normoglicemia está associada a menores taxas de infecções e de falências orgânicas e, conseqüentemente, a menor mortalidade ${ }^{10}$.

Para a implementação do controle glicêmico intensivo é recomendável que se disponha de um protocolo com estratégias definidas para manter a glicemia na faixa escolhida e para prevenir e tratar as complicações, principalmente a hipoglicemia. Entre os benefícios de uma abordagem protocolizada citam-se a aplicação de melhores práticas baseadas em evidências, a padronização do tratamento, a redução da variabilidade no atendimento, a redução de complicações e melhor desfecho clínico ${ }^{22}$.

O protocolo de Yale foi selecionado para uniformizar o controle glicêmico na UTI do Hospital de Caridade de Florianópolis por contar com instruções detalhadas e objetivas de manuseio e também por adotar uma faixa glicêmica considerada mais segura, do que a faixa de 80 a $110 \mathrm{mg} / \mathrm{dL}$, preconizada por Van den Berghe

Tabela 2 - Resultados do Controle Glicêmico nos Grupos Estudados

\begin{tabular}{|c|c|c|c|}
\hline Variáveis & $\mathrm{GC}(\mathrm{n}=60)$ & $\mathrm{GP}(\mathrm{n}=52)$ & Valor de $p$ \\
\hline $\mathrm{N}^{\circ}$ de glicemias diárias & $5,4 \pm 1,2$ & $14,8 \pm 4,2$ & $<0,001$ \\
\hline $\begin{array}{l}\text { Mediana em horas para glicemia } \leq 140 \mathrm{mg} / \mathrm{dL} \\
\text { (intervalo } 25 \%-75 \% \text { ) }\end{array}$ & $96(46-278)$ & $7(4-10)$ & $<0,001$ \\
\hline $\begin{array}{l}\text { Glicemias } 80-140 \mathrm{mg} / \mathrm{dL} \\
\text { (\% mensurações) }\end{array}$ & $799(32 \%)$ & 3527 (65\%) & $<0,001$ \\
\hline $\begin{array}{l}\text { Glicemias } \leq 60 \mathrm{mg} / \mathrm{dL} \\
\text { Pacientes (\%) }\end{array}$ & $13(21,6 \%)$ & $22(42,3 \%)$ & $<0,005$ \\
\hline $\begin{array}{l}\text { Glicemias } \leq 40 \mathrm{mg} / \mathrm{dL} \\
\text { Pacientes (\%) }\end{array}$ & $2(3,3 \%)$ & $4(7,7 \%)$ & ns \\
\hline $\begin{array}{l}\text { Glicemia média durante } \\
\text { a insulinoterapia }\end{array}$ & $181,70 \pm 36,05$ & $131,21 \pm 14,72$ & $<0,001$ \\
\hline
\end{tabular}


e col. ${ }^{10}$. O presente estudo avaliou a efetividade e a segurança da insulinoterapia venosa contínua administrada conforme este protocolo. Os resultados demonstram que ele foi efetivo e melhorou significativamente o controle glicêmico numa população heterogênea de pacientes críticos clínicos e cirúrgicos, comparativamente ao controle glicêmico antes praticado.

Após a implantação do protocolo, a glicemia média foi reduzida de 181,7 $\pm 36,1 \mathrm{mg} / \mathrm{dL}$ para 131,2 $\pm 14,1 \mathrm{mg} / \mathrm{dL}$ e também houve um aumento significativo na proporção das glicemias dentro da faixa-alvo que subiu de $32 \%$ para $65 \%$. O tempo necessário para as glicemias atingirem a faixa de controle também diminuiu significativamente.

A hipoglicemia é considerada a principal complicação associada a insulinoterapia venosa contínua e ocorre em torno de $4 \%$ a $7 \%$ dos pacientes. Ela pode causar manifestações adrenérgicas como sudorese, taquicardia, ansiedade, palpitações e manifestações neuroglicopênicas como cefaléia, confusão mental, convulsões e coma. Nos pacientes críticos a detecção dos episódios hipoglicêmicos fica dificultada nos inconscientes e naqueles em ventilação mecânica. São fatores de risco para o desenvolvimento de hipoglicemia; protocolos de insulinoterapia venosa contínua com faixas estreitas de controle glicêmico, insuficiência renal aguda com necessidade de hemodiálise, sepse, utilização de fármacos vasoativos, corticóides e qualquer redução na administração de terapia nutricional sem redução concomitante na taxa de infusão da insulina ${ }^{21}$. Um protocolo de manuseio adequado deve incluir determinações freqüentes de glicemia, recomendações específicas para alterar as taxas de infusão e exigir administração imediata de glicose hipertônica na vigência de um evento hipoglicêmico ${ }^{16}$.

Neste estudo o protocolo utilizado pode ser considerado seguro pois, embora tenha se observado um aumento estatisticamente significativo na taxa de hipoglicemia total no GP, não houve aumento significativo na taxa de hipoglicemia grave comparativamente ao período anterior. Os episódios de hipoglicemia foram prontamente detectados e corrigidos pela administração venosa de glicose a $50 \%$ de acordo com as instruções do protocolo. Também não se observou manifestações clínicas adversas ou complicações relacionadas aos eventos hipoglicêmicos. O percentual de pacientes que apresentou hipoglicemia grave é comparável com aqueles encontrados por Zimmerman e col. ${ }^{19}$ em UTI cardiotorácica e por Vriesendorp e col. ${ }^{23} \mathrm{em}$ UTI geral clínica e cirúrgica. Os resultados observados com o controle glicêmico intensivo no presente estudo, numa população mista de pacientes críticos clínicos e cirúrgicos, aproximam-se bastante dos resultados encontrados por Goldberg e col. no estudo original que descreveu o protocolo de Yale e que foi realizado numa população exclusiva de pacientes clínicos ${ }^{18}$. Esses autores encontraram uma mediana de tempo de nove horas para atingir a faixa glicêmica de 80 a 140 mg/dL em comparação com sete horas no atual estudo. A proporção de glicemias dentro da faixa foi similar: $66 \%$ no estudo de Yale e $65 \%$ neste estudo. A taxa de infusão horária de insulina venosa foi $50 \%$ menor no nosso estudo (mediana 2 unidades/hora versus 4 unidades/hora) porém os pacientes receberam infusão de insulina por período mais prolongado (mediana 156 horas versus 61 horas). Quanto à ocorrência de hipoglicemia grave, foram três episódios na casuística de Goldberg e col. e quatro na atual. Até onde se pode verificar este é o primeiro estudo a relatar o desempenho do protocolo de Yale numa realidade assistencial diferente daquela dos estudos publicados por Goldberg e col. ${ }^{18,24-25}$.

Dentre as limitações do presente estudo salienta-se o fato de ser um levantamento retrospectivo, portanto sujeito a viés na coleta dos dados. Também a comparação entre dois períodos diferentes pressupõe que as práticas terapêuticas tenham sido similares. Apesar de não ter sido detectada nenhuma mudança nas práticas na unidade durante estes períodos, que não a implantação do controle glicêmico intensivo, não se pode afastar a influência do efeito Hawthorne, devido à utilização do próprio protocolo.

Ainda existem muitas dúvidas a respeito do controle glicêmico intensivo nos pacientes críticos para serem esclarecidas. A interrupção de um estudo multicêntrico alemão sobre sepse grave, devido à elevada incidência de hipoglicemia associada à insulinoterapia intensiva ${ }^{26}$ e a constatação, no estudo recente de Van den Berghe e col. em pacientes clínicos ${ }^{27}$, de que a taxa de mortalidade foi maior nos pacientes sob controle glicêmico intensivo que permaneceram menos de três dias na UTI motivaram vários debates ${ }^{28-32}$. Entre outros tópicos discute-se que o controle estrito deve ser aplicado indistintamente a todos os pacientes que apresentam elevações da glicemia, se existem faixas diferentes de controle glicêmico para diferentes doenças e populações de pacientes críticos. Espera-se que dois grandes estudos multicêntricos em andamento; NICE-SUGAR que estuda 4.500 pacientes clínicos e cirúrgicos em UTI na Austrália, Nova Zelândia e Canadá e GLUControl que avalia 3.500 pacientes clínicos e cirúrgicos em vários centros na Europa em breve respondam estas 
indagações ${ }^{28,29}$. Na opinião de vários especialistas ${ }^{28,30-}$ ${ }^{32}$, até que se tenham mais respostas, é uma atitude prudente adotar regimes de controle glicêmico menos agressivos $^{33}$, mas ainda comprovadamente efetivos ${ }^{34}$, que assegurem glicemias abaixo de $150 \mathrm{mg} / \mathrm{dL}$, principalmente em UTI com menor disponibilidade de recursos humanos.

Concluindo, este estudo demonstrou que o protocolo de Yale foi efetivo e seguro para manusear a insulinoterapia por via venosa contínua, visando o controle glicê- mico estrito de pacientes críticos clínicos e cirúrgicos em um hospital comunitário brasileiro.

\section{AGRADECIMENTOS}

À Equipe de Enfermagem do Serviço de Terapia Intensiva do Hospital de Caridade de Florianópolis cuja participação foi e continua sendo fundamental para o bom desempenho deste e dos demais protocolos de tratamento utilizados no serviço.

Anexo 1 - Protocolo de Infusão de Insulina de Yale

Este protocolo de infusão de insulina por via venosa é destinado a pacientes adultos críticos com hiperglicemia, em UTI, mas não é especificamente talhado para pacientes com emergências diabéticas tais como ceto-acidose diabética e estados hiperosmolares. Quando a glicemia estiver acima de $500 \mathrm{mg} / \mathrm{dL}$ ou com respostas inesperadas à infusão de insulina ou em situações que não se enquadrem nestas recomendações, comunicar o médico intensivista.

Iniciando uma infusão de insulina:

1. Infusão de Insulina: $50 \mathrm{UI}$ de insulina regular mais $50 \mathrm{~mL}$ de solução fisiológica a $0,9 \%$ (1 UI = $1 \mathrm{~mL})$, administrar em bomba de seringa em incrementos de $0,5 \mathrm{U} / \mathrm{h}$.

2. PRIMING: descartar os primeiros $5 \mathrm{~mL}$ sempre que trocar o equipo perfusor (para saturar os locais de ligação de insulina no equipo).

3. Faixa Alvo de Glicemia: $80-140 \mathrm{mg} / \mathrm{dL}$

4. Bolus e Taxa de Infusão Inicial de Insulina: dividir a glicemia inicial por 100 e arredondar para o 0,5 U mais próximo para o bolus e taxa de infusão inicial. Exemplos:

1) HGT inicial $=325 \mathrm{mg} / \mathrm{dL}: 325 \div 100=3,25$ arredondando para mais $=3,5$ : bolus venoso de $3,5 \mathrm{U}(3,5 \mathrm{ml})$ e inicia a infusão a 3,5 $\mathrm{U} / \mathrm{h}(3,5 \mathrm{~mL} / \mathrm{h})$

2) $\mathrm{HGT}$ inicial $=174 \mathrm{mg} / \mathrm{dL}: 174 \div 100=1,74$ arredondando para menos 1,5 : bolus venoso de $1,5 \mathrm{U}(1,5 \mathrm{~mL})$ e inicia infusão a $1,5 \mathrm{U} / \mathrm{h}$ $(1,5 \mathrm{~mL} / \mathrm{h})$

Monitorização da Glicemia (HGT)

1. Medir a HGT de $1 / 1$ hora até estabilizada (3 valores consecutivos dentro da faixa alvo). Em pacientes hipotensos a glicemia capilar pode ser inacurada e é aceitável amostra obtida de cateteres vasculares.

2. Então medir HGT de 2/2 horas; se estável por 12-24 horas HGT pode ser espaçado para 4/4 horas SE:

a) Sem alteração significativa na condição clínica; e b). sem alteração significativa no aporte nutricional

3) Se ocorrer um dos seguintes, considerar o reinício horário do HGT até estar estabilizado novamente (2 a 3 valores dentro da faixa):

a) Qualquer alteração na taxa de infusão da insulina (HGT fora da faixa alvo);

b) Alterações significativas na condição clínica;

c) Início ou interrupção de vasopressores ou corticosteróides;

d) Início ou interrupção de terapia dialítica renal (hemodiálise, etc.);

e) Início, interrupção ou modificação de terapia nutricional (NPT ou NE);

Alterando a Taxa de Infusão de Insulina:

Se HGT < 50 mg/dL: PARAR A INFUSÃO DE INSULINA

1. Administrar $25 \mathrm{~g}$ de glicose por via venosa ( $50 \mathrm{~mL}$ glicose a $50 \%$ ) e medir HGT cada 15 minutos

2. Quando HGT $\geq 100 \mathrm{mg} / \mathrm{dL}$, aguardar 1 hora e reiniciar a infusão da insulina a $50 \%$ da taxa de infusão original

Se HGT 50-74 mg/dL: PARAR A INFUSÃO DE INSULINA

1. Se sintomático (ou impossível de avaliar) administrar $25 \mathrm{~g}$ de glicose por via venosa e medir HGT cada 15 minutos

2. Se assintomático administrar $12,5 \mathrm{~g}$ de glicose por via venosa ou $250 \mathrm{~mL}$ de sucos por via oral e medir HGT cada 15-30 minutos

3. Quando HGT $\geq 100 \mathrm{mg} / \mathrm{dL}$, aguardar 1 hora e reiniciar a infusão da insulina a $75 \%$ da taxa de infusão original

SE HGT > $75 \mathrm{mg} / \mathrm{dL}$ :

PASSO 1: Determinar o VALOR ATUAL DO HGT - identificar uma coluna na tabela:

$$
\begin{array}{|l|l|l|l|}
\text { HGT 75-99 mg/dL } & \text { HGT } 100-139 \mathrm{mg} / \mathrm{dL} & \text { HGT } 140-199 \mathrm{mg} / \mathrm{dL} & \text { HGT } \geq 200 \mathrm{mg} / \mathrm{dL} \\
\hline
\end{array}
$$


PASSO 2: Determinar a TAXA DE VARIAC̣ÃO à partir do HGT prévio - identificar uma CÉLULA na tabela - Ir para a coluna das INSTRUÇÕES

(Nota: se o último HGT foi medido 2-4 horas antes do atual, calcular a taxa horária de variação. Exemplo: Se HGT as $14 \mathrm{~h}$ foi 150 mg/ dL e as 16 h é agora $120 \mathrm{mg} / \mathrm{dL}$, a variação total nas 2 horas é - $30 \mathrm{mg} / \mathrm{dL}$; porém a variação horária é $-30 \div 2$ horas = -15 mg/dL/h)

\begin{tabular}{|c|c|c|c|c|}
\hline HGT $75-99 \mathrm{mg} / \mathrm{dL}$ & HGT $100-139 \mathrm{mg} / \mathrm{dL}$ & HGT $140-199$ mg/dL & $\mathrm{HGT} \geq 200 \mathrm{mg} / \mathrm{dL}$ & INSTRUÇÕES* \\
\hline & & $\mathrm{HGT} \uparrow>50 \mathrm{mg} / \mathrm{dL} / \mathrm{h}$ & HGT $\uparrow$ & 个 INFUSÃO POR “2 $\mathbf{\Delta}$ " \\
\hline & $\mathrm{HGT} \uparrow>25 \mathrm{mg} / \mathrm{dL} / \mathrm{h}$ & $\begin{array}{c}\text { HGT } \uparrow 1-50 \mathrm{mg} / \mathrm{dl} / \mathrm{h} \\
\text { ou INALTERADO }\end{array}$ & $\begin{array}{c}\text { INALTERADO } \\
\text { ou HGT } \downarrow 1-25 \mathrm{mg} / \mathrm{dL} / \mathrm{h}\end{array}$ & 个 INFUSÃO POR “॥” \\
\hline HGT $\uparrow$ & $\begin{array}{l}\text { HGT } \uparrow 1-25 \mathrm{mg} / \mathrm{dL} / \mathrm{h} \\
\text { HGT INALTERADO ou } \\
\text { HGT } \downarrow 1-25 \mathrm{mg} / \mathrm{dL} / \mathrm{h}\end{array}$ & HGT $\downarrow 1-50 \mathrm{mg} / \mathrm{dL} / \mathrm{h}$ & HGT $\downarrow 26-75 \mathrm{mg} / \mathrm{dL} / \mathrm{h}$ & NÃO ALTERA INFUSÃO \\
\hline $\begin{array}{l}\text { INALTERADO } \\
\text { ou HGT } \downarrow 1-25 \mathrm{mg} / \mathrm{dL} / \mathrm{h}\end{array}$ & HGT $\downarrow 26-50 \mathrm{mg} / \mathrm{dL} / \mathrm{h}$ & HGT $\downarrow 51-75 \mathrm{mg} / \mathrm{dL} / \mathrm{h}$ & HGT $\downarrow 76-100 \mathrm{mg} / \mathrm{dL} / \mathrm{h}$ & $\downarrow$ INFUSÃO POR “॥” \\
\hline $\begin{array}{l}\text { HGT } \downarrow>25 \mathrm{mg} / \mathrm{dL} / \mathrm{h} \\
\text { Ver abaixo\# }\end{array}$ & $\mathrm{HGT} \downarrow>50 \mathrm{mg} / \mathrm{dLI} / \mathrm{h}$ & $\mathrm{HGT} \downarrow>75 \mathrm{mg} / \mathrm{dL} / \mathrm{h}$ & $\mathrm{HGT} \downarrow>100 \mathrm{mg} / \mathrm{dL} / \mathrm{h}$ & $\begin{array}{c}\text { INTERROMPE } 30 \text { min' } \\
\text { depois } \\
\downarrow \text { INFUSÃO POR “2 } \mathbf{~} \text { ” }\end{array}$ \\
\hline
\end{tabular}

\# interrompe infusão insulina, mede HGT 30/30 min: quando HGT $\geq 100$ mg/dl, reinicia infusão com 75\% da taxa anterior.

*ALTERAÇÕES NA TAXA DE INFUSÃO (“॥”) são determinadas pela taxa atual:

\begin{tabular}{|c|c|c|}
\hline $\begin{array}{c}\text { Taxa atual } \\
(\mathrm{U} / \mathrm{h})\end{array}$ & $\begin{array}{c}\boldsymbol{\Delta}=\text { alteração na taxa } \\
(\mathrm{U} / \mathrm{h})\end{array}$ & $\begin{array}{c}2 \boldsymbol{\Delta}=2 \times \\
\text { alteração na taxa } \\
(\mathrm{U} / \mathrm{h})\end{array}$ \\
\hline$<3$ & 0,5 & 1 \\
\hline $3,0-6,0$ & 1 & 2 \\
\hline $6,5-9,5$ & 1,5 & 3 \\
\hline $10-14,5$ & 2 & 4 \\
\hline $15-19,5$ & 3 & 6 \\
\hline $20-24,5$ & 4 & 8 \\
\hline$\geq 25$ & $\geq 5$ & 10 (consultar MD) \\
\hline
\end{tabular}

\section{REFERÊNCIAS}

01. Coursin DB, Connery LE, Ketzler JT - Perioperative diabetic and hyperglycemic management issues. Crit Care Med, 2004;32:(Suppl4):S116S125.

02. Krinsley JS - Association between hyperglycemia and increased hospital mortality in a heterogenous population of critically ill patients. Mayo Cli Proc, 2003;78:1471-1478.

03. Malmberg K, Norhammar A, Wedel H et al - Glycometabolic state at admission: important risk marker of mortality in conventionally treated patients with diabetes mellitus and acute myocardial infarction: longterm results from the Diabetes and Insulin-Glucose Infusion in Acute Myocardial Infarction (DIGAMI) study. Circulation, 1999;99:2626-2632.

04. Capes SE, Hunt D, Malmberg K et al - Stress hyperglycaemia and increased risk of death after myocardial infarction in patients with and without diabetes: a systematic overview. Lancet, 2000;355:773-778.

05. Capes SE, Hunt D, Malmberg $\mathrm{K}$ et al - Stress hyperglycemia and prognosis of stroke in nondiabetic and diabetic patients: a systematic overview. Stroke, 2001;32:2426-2432.

06. Yendamuri S, Fulda GJ, Tinkoff GH - Admission hyperglycemia as a prognostic indicator in trauma. J Trauma, 2003;55:33-38.

07. Rassias AJ, Marrin CA, Arruda $\mathrm{J}$ et al - Insulin infusion improves neutrophil function in diabetic cardiac surgery patients. Anesth Analg, 1999;88:1011-1016.

08. Golden $\mathrm{SH}$, Peart-Vigilance $\mathrm{C}, \mathrm{Kao} \mathrm{WH}$ et al - Perioperative glycemic control and the risk of infectious complications in a cohort of adults with diabetes. Diabetes Care, 1999;22:1408-1414.

09. Dandona P, Mohanty P, Chaudhuri A et al - Insulin infusion in acute illness. J Clin Invest, 2005;115:2069-2072.

10. van den Berghe $\mathrm{G}$, Wouters $\mathrm{P}$, Weekers $\mathrm{F}$ et al - Intensive insulin therapy in critically ill patients. N Engl J Med, 2001;345:1359-1367.

11. American College of Endocrinology Task Force on Inpatient Diabetes and Metabolic Control - Position Statement on Inpatient Diabetes and Metabolic Control. Endocr Pract, 2004;10:78-82.

12. Marik PE, Raghavan M - Stress-hyperglycemia, insulin and immunomodulation in sepsis. Intensive Care Med, 2004;30:748-756.

13. Cariou A, Vinsonneau C, Dhainaut JF - Adjunctive therapies in sepsis: an evidence-based review. Crit Care Med, 2004;32:(Suppl11):S562-S570.

14. Dellinger RP, Carlet JM, Masur $\mathrm{H}$ et al - Surviving Sepsis Campaign guidelines for management of severe sepsis and septic shock. Crit Care Med, 2004;32:858-873.

15. Brown G, Dodek P - Intravenous insulin nomogram improves blood glucose control in the critically ill. Crit Care Med, 2001;29:1714-1719.

16. Bode BW, Braithwaite SS, Steed RD et al - Intravenous insulin infusion therapy: indications, methods, and transition to subcutaneous insulin therapy. Endocr Pract, 2004;10:(Suppl2):S71-S80.

17. Kanji S, Singh A, Tierney $M$ et al - Standardization of intravenous insulin therapy improves the efficiency and safety of blood glucose control in critically ill adults. Intensive Care Med, 2004;30:804-810.

18. Goldberg PA, Siegel MD, Sherwin RS et al - Implementation of a safe and effective insulin infusion protocol in a medical intensive care unit. Diabetes Care, 2004;27:461-467.

19. Zimmerman CR, Mlynarek ME, Jordan JA et al - An insulin infusion pro- 


\section{AVALIAÇÃO DA EFETIVIDADE E SEGURANÇA DO PROTOCOLO DE INFUSÃO DE INSULINA DE YALE PARA O CONTROLE GLICÊMICO INTENSIVO}

tocol in critically ill cardiothoracic surgery patients. Ann Pharmacother, 2004;38:1123-1129.

20. Chant C, Wilson G, Friedrich JO - Validation of an insulin infusion nomogram for intensive glucose control in critically ill patients. Pharmacotherapy, 2005;25:352-359.

21. Nasraway SA Jr - Hyperglycemia during critical illness. JPEN J Parenter Enteral Nutr, 2006;30:254-258.

22. Morris AH - Treatment algoritms and protocolized care. Curr Opin Crit Care, 2003;9:236-240.

23. Vriesendorp TM, van Santen S, DeVries JH et al - Predisposing factors for hypoglycemia in the intensive care unit. Crit Care Med, 2006;34:96-101.

24. Goldberg PA, Sakharova OV, Barrett PW et al - Improving glycemic control in the cardiothoracic intensive care unit: clinical experience in two hospital settings. J Cardiothorac Vasc Anesth, 2004;18;690-697.

25. Goldberg PA, Roussel M, Inzucchi SE - Clinical results of an updated insulin infusion protocol in critically ill patients. Diabetes Spectrum, 2005;18:188-191.

26. Brunkhorst FM, Kuhnt E, Engel $\mathrm{C}$ et al - Intensive insulin therapy in patients with severe sepsis and septic shock is associated with an increa- sed rate of hypoglycemia - results from a randomized multicenter study. Infection, 2005;33:(Suppl1):19-20.

27. Van den Berghe $\mathrm{G}$, Wilmer A, Hermans $\mathrm{G}$ et al - Intensive insulin therapy in the medical ICU. N Engl J Med, 2006;354:449-461.

28. Angus DC, Abraham E - Intensive insulin therapy in critical illness. Am J Respir Crit Care Med, 2005;172:1358-1359.

29. Bellomo R, Egi M - Glycemic control in the intensive care unit: why we should wait for NICE-SUGAR. Mayo Clin Proc, 2005;80:1546-1548.

30. Malhotra A - Intensive insulin in intensive care. N Engl J Med, 2006;354;516-518.

31. Polderman $\mathrm{KH}$, Girbes AR - Intensive insulin therapy: of harm and health, of hypes and hypoglycemia. Crit Care Med, 2006;34:246-248.

32. Watkinson P, Barber VS, Young JD - Strict glucose control in the critically ill. BMJ, 2006;332:865-866.

33. Krinsley JS - Effect of an intensive glucose management protocol on the mortality of critically ill adult patients. Mayo Clin Proc, 2004;79:9921000.

34. Finney SJ, Zekveld C, Elia A et al - glucose control and mortality in critically ill patients. JAMA, 2003;290:2041-2047. 